\title{
REPRESENTACIONES SOBRE LA EVALUACIÓN DE ESTUDIANTES EN FORMACIÓN INICIAL DOCENTE ${ }^{1}$
}

\author{
Ana Carolina Maldonado Fuentes* \\ Universidad del Bío-Bío, Chile \\ amaldonado@ubiobio.cl \\ Pedro Rodrigo Sandoval Rubilar** \\ Universidad del Bío-Bío, Chile \\ psandoval@ubiobio.cl
}

Recibido: 16/08/2019 Aceptado: 18/11/2019

\section{Resumen}

Evaluar es una tarea clave de la función docente en el campo Educativo. No obstante, estudios empíricos evidencian su carácter polisémico y confuso, siendo compleja de representar para el profesorado; quienes, tras haber sido evaluados en su trayectoria escolar, enfrentan el desafío de abordar la evaluación como objeto de aprendizaje durante su formación profesional. En este contexto, resulta de interés identificar las representaciones que han construido estudiantes en Formación Inicial Docente sobre la evaluación. Desde un enfoque cualitativo, este estudio, de carácter interpretativo, da cuenta de cómo, para qué y qué efectos tiene evaluar, a partir de textos elaborados por 51 estudiantes de Pedagogía en Educación Básica de una universidad estatal chilena. Al categorizar más de cien unidades de análisis por pregunta con N-Vivo 12, se confirma la premisa de que coexisten diferentes significados de la evaluación en los textos. Además, se observa escasa alusión a los fundamentos de la evaluación educativa y una tendencia a replicar el discurso del sentido común propio de la escolarización. Dicho hallazgo podría indicar que la experiencia previa a la Formación Inicial incidiría fuertemente en la representación de la evaluación en los futuros profesores, pese a estar cursando estudios formales para la docencia.

Palabras clave: Formación Inicial Docente - Evaluación del Aprendizaje - Evaluación Educativa Representaciones - Profesorado.

\section{Abstract}

Assessment is a key component of the learning process; however, empirical research has shown evidence of its polysemous and complex character. Teachers' representations about assessment are difficult to construct, as they face the challenge of covering it as a learning goal during their

\footnotetext{
${ }^{1}$ Se agradece el apoyo recibido para la realización de este trabajo por parte de la Comisión Nacional de Investigación Científica y Tecnológica, Chile. Financia CONICYT-PFCHA/Doctorado Nacional/2017-21170257. Doctorado en Educación en Consorcio-Universidad Católica de la Santísima Concepción (UCSC).

* Profesora de Castellano (1993), Licenciada en Estética (1993) y Magíster en Ciencias de la Educación (1999). Profesora de lenguaje en el sistema escolar chileno (1995 a 2007), de español como lengua extranjera en la P. Universidad Católica de Chile (1997 a 2007) y de formación docente en la Universidad Academia de Humanismo Cristiano (UAHC) (2004 a 2007). Académica en la Universidad del Bío-Bío desde 2008 a la fecha. Jefa de la Unidad de Gestión Curricular y Monitoreo de la Universidad del Bío-Bío (2010-2014).

**Doctor en Ciencias de la Educación. Académico, investigador y docente de Pregrado, Magíster y Doctorado en distintas universidades de Chile. Actualmente académico y representante de Posgrado de la Facultad de Educación y Humanidades en el Comité de Postgrado de la Universidad del Bío-Bío.
} 
undergraduate studies but they have been the object of assessment throughout their primary and secondary studies. In this context, interest about representations of assessment constructed by Initial Teacher Training students was aroused. From a qualitative approach, this interpretative study explains how students should be assessed, for what purpose and which effects are produced on them. Texts written by 51 Primary Teaching students at a public university in Chile were analysed. After classifying over a hundred units of analysis per question with N-Vivo 12, the premise that different meanings of assessment coexist in the texts was confirmed. Scarce mention of the theoretical basis for assessment was also observed and a tendency to replicate the common sense discourse of schooling was noticed. This result could show that Primary Teaching students' experiences before Initial Teacher training could strongly influence representations of assessment constructed by them, even if they are receiving formal education to become teachers.

Keywords: Initial Teacher Training - Assessment - Educational Evaluation - Representations Teachers.

\section{Introducción}

En las últimas décadas, la formación del profesorado como docente Evaluador ha cobrado relevancia, existiendo un discurso consensuado en torno a la idea de que los maestros constituyen un factor fundamental en el mejoramiento de los resultados de aprendizaje de los estudiantes. Inclusive, se ha llegado a afirmar que "la calidad de un sistema educativo tiene como techo la calidad de sus docentes" (Barber y Moushed, 2008, p.15); siendo relevante la figura del profesor y su preparación para evaluar, considerada como una de las tareas primordiales de su ejercicio profesional docente (Black y Wiliam, 1998).

En coherencia, las políticas públicas han sido diligentes en proveer una serie de estrategias para fortalecer la formación del profesorado de manera sostenida en el tiempo, cuyos hitos son reconocidos en Chile, sobre todo, tras la implementación de la Reforma Educativa en los años noventa (1997-2018). En particular, a nivel de discurso se ha invitado a poner el foco en el aprendizaje, acorde al propósito de evaluar durante el proceso, lo que se evidencia en normativas y marcos curriculares para el sistema escolar; junto con explicitar orientaciones para la Formación Inicial Docente y el ejercicio docente en aula, entre otros.

Sin embargo, pese a ser un tema desafiante del desarrollo profesional docente, la formación para llegar a ser un evaluador ha sido descrita de modo indirecto en la literatura especializada. Por un lado, dado que el mismo concepto de evaluación es un constructo que ha ido cambiando a través del tiempo (De Camilloni, 1998; Escudero, 2003; Moreno Olivos, 2014; Santos Guerra, 2014); y, por otro, en virtud de lo complejo que resulta abordar empíricamente los distintos elementos intervinientes en el ejercicio de la tarea de evaluación, tales como las relaciones de autoridad, la representación del poder y la exclusión al interior de la escuela (Xu y Liu, 2009).

En esta misma línea, estudios sobre el pensamiento del profesor invitan a indagar en las concepciones, creencias o representaciones que podrían estar incidiendo en la enseñanza (Kelly, 1995; Jackson, 1998). Al respecto, la teoría de las Representaciones Sociales ha resultado altamente explicativa, pues permite la identificación de elementos de sentido común, que son compartidos por grupos de profesores y se naturalizan de manera espontánea tanto en el discurso como en la práctica pedagógica (Salinas et al., 2006; Aguilar et al. 2011).

El supuesto que orienta estos trabajos es la existencia de elementos implícitos en la actuación del profesorado, cuya construcción expresa sentidos, sistemas de anticipación y expectativas elaboradas en conjunto y en interacción social. De allí que, sea difícil sostener que el profesorado logre apropiarse de manera mecánica de las premisas normativas reguladoras de la práctica, ya que todo escenario educativo "se imbrica de un carácter sistémico y de elementos simbólicos" (Gutiérrez, 2016, p.49) que dan origen a marcos de referencia comunes, los cuales orientan y dan sentido al accionar.

No obstante, a pesar del creciente interés por el estudio de las representaciones del profesorado, son aún insuficientes los trabajos en el campo de la evaluación. En concreto, indagaciones sobre la Formación Inicial Docente (en adelante FID), realizados sobre futuros profesores de Educación Básica chilenos, han concluido que es necesario disponer de una mayor cantidad de estudios sobre didáctica, currículum y evaluación (Agencia de Calidad de la Educación, 2016). Dichos hallazgos complementan antecedentes previos sobre docentes en ejercicio a nivel país, que dan cuenta de deficiencias en lo referido a las prácticas evaluativas y su aplicación en el sistema escolar (Manzi, González y Sun, 2011); de lo que se desprende que la formación del docente de Educación Básica en este ámbito es una preocupación para las instituciones educativas.

Lo anterior, invita a poner mayor atención a las representaciones elaboradas por los futuros profesores respecto de la evaluación y a la preparación recibida para ejercer dicha tarea, pues es sabido que para que el profesorado pueda desarrollar habilidades en su futuro profesional requiere haberlas vivenciado 
REVISTA DE LA ESCUELA DE CIENCIAS DE LA EdUCACIÓN, AÑO 16, NRO. 15, VOL. 2, JULIO A DICIEMRE DE 2020. PÁGINAS 119-130. ISSN 2362-3349 (EN LíNEA). REPRESENTACIONES SOBRE LA EVALUACIÒN DE ESTUDIANTES EN FORMACIÒN INCIAL DOCENTE ANA CAROLINA MALDONADO FUENTES. PEDRO RODRIGO SANDOVAL RUBILAR.

durante su formación (Comellas, 2000). Conocer en mayor profundidad dichas representaciones, nos permitirá enfrentar de mejor manera la formación del profesorado de Educación Básica como evaluador. Por tanto, la finalidad del presente estudio es caracterizar las representaciones respecto de la tarea de evaluar (cómo y para qué se evalúa) que han construido estudiantes de Pedagogía en Educación Básica durante su Formación Inicial Docente, a partir de relatos elaborados como futuro profesor al término del primer ciclo formativo. Para ello, a continuación, se exponen argumentos en torno a la relevancia de la evaluación como tarea docente y del estudio de las representaciones sociales. Con posterioridad, se aborda la metodología, especificando los participantes y las decisiones tomadas para el análisis de información. Luego se realiza una descripción general de los resultados, finalizando con la discusión, y las conclusiones.

\section{Importancia de la evaluación}

La evaluación constituye parte indispensable y constitutiva de la enseñanza y el aprendizaje, siendo central en la comprensión, regulación y mejoramiento de estos procesos. Asimismo, puede ser entendida como "un componente didáctico del proceso de enseñanza-aprendizaje y, a la vez, una actividad reguladora imprescindible y una vía para mejorar el aprendizaje del estudiantado y contribuir a su desarrollo integral" (Hernández-Nodarse, 2017, p. 2), por lo que ha llegado a sostenerse que "ninguna innovación didáctica será efectiva si no va acompañada de innovaciones en el modo de concebir la evaluación" (López Pastor y Palacios Picos, 2012, p. 320). En consecuencia, indagar en este ámbito resulta indispensable al momento de aspirar al mejoramiento de la calidad de la Educación.

En este marco, es preciso explicitar que desde hace más de una década se ha promovido la implementación de un modelo teórico alternativo de evaluación en el sistema chileno, caracterizado por el uso de distintas estrategias para la evaluación y retroalimentación durante todo el proceso (Himmel el al., 1999; Ahumada, 2005). Más aún, recientemente se ha instado de modo explícito a la evaluación formativa en el aula (Förster, 2017; MINEDUC, 2018), invitando al profesorado a integrar el uso pedagógico de la evaluación en la enseñanza. Por tanto, cabría suponer que la evaluación, en general, y la evaluación formativa, en particular, son puntos fuertes de la formación de profesores. Sin embargo, investigaciones han puesto en evidencia cómo la experiencia escolar y universitaria ha estado marcada por un uso técnico e instrumental de la evaluación, bajo el supuesto de que por medio de la calificación es posible medir el aprendizaje. Esto se traduce, por ejemplo, en la preocupación por mejorar la calidad en el diseño de diversos instrumentos evaluativos, tales como pruebas, pautas de evaluación y rúbricas; tema que se ha documentado como de alto interés durante la formación continua de los docentes.

Por añadidura, la evaluación es uno de los cinco problemas más comunes del profesorado novel y en ejercicio (Veeman en 1984, citado por Mercado Cruz, 2013, p.98), y, al mismo tiempo, representa un ámbito de desempeño que resulta paradójico en la práctica dado que, pese a la intención de evaluar aprendizajes, la mayoría de las instancias de evaluación que se implementan en el ejercicio profesional docente son de corte sumativo. En Chile esto ha sido visualizado en el hecho que "los profesores están más familiarizados con la forma tradicional de evaluación de los estudiantes" (Förster, 2017, p. 97), en coherencia con la normativa que regula el sistema de evaluación y calificación escolar. Este último se caracteriza por responder a la función social de la evaluación, ajustándose al sentido último de promoción y certificación de los aprendizajes.

En síntesis, a nivel teórico, empírico y normativo, se observa cómo la evaluación es uno de los ámbitos más desafiantes de la profesión, de lo que se infiere que la preparación para el ejercicio profesional como evaluador constituye uno de los nudos críticos de la formación del profesorado. Al mismo tiempo, puede representarse como un concepto confuso y controversial, al coexistir en el discurso educativo variadas definiciones y enfoques, las que no necesariamente son explícitas o sometidas a reflexión. En particular, los estudios analizados invitan a profundizar en lo que sucede durante la FID, debido a que, siguiendo a Ferry (2016) la formación docente puede ser entendida como una secuencia de etapas, cuyo rasgo distintivo consiste en que el sujeto se forma así mismo en una dinámica de mediaciones que le permiten ponerse en condiciones de ejercer una determinada práctica profesional. Entre ellas destaca la representación de trabajo a realizar, las concepciones e imágenes asociadas a su desempeño, a partir de lo cual el futuro profesor tiene la posibilidad de apropiarse del rol docente en forma previa al ejercicio de la profesión. Dentro de lo cual, la tarea de evaluar, resulta un ámbito de interés.

\section{Representaciones sociales acerca de la evaluación}

Tal como se señala en el apartado de antecedentes, el estudio del pensamiento del profesor encuentra en la teoría de las Representaciones Sociales (en adelante RS) un marco referencial pertinente para indagar sobre los elementos implícitos de la actuación docente. El supuesto que subyace es que la actividad (en este caso, el comportamiento observado del profesorado) se relaciona con elementos cognitivos, afectivos, valorativos y sociales, generalmente implícitos, cuya elaboración sucede de manera mediatizada por interacciones con otros, con quienes se comparten significados y sentidos, pudiendo interpretar y desenvolverse en formas de actuación naturalizadas. Por ejemplo, "los futuros maestros asumen como algo 
natural que lo que deben hacer es enseñar" (Cárcamo, 2015, p. 272). De allí que sea factible aludir a las representaciones como un conocimiento espontáneo, ingenuo, de sentido común o pensamiento natural.

El concepto de Representaciones Sociales fue formulado originalmente por Serge Moscovici (19252014) en los años 60', a fin de distinguir el conocimiento científico de aquel basado en el sentido común; el cual, en vez de buscar la verdad, se propone alcanzar una mejor comprensión entre los sujetos. Desde el punto de vista epistemológico, se retoma la tradición durkhiana para dar sentido a un tipo de representación social de carácter dinámico, que es individual y colectiva, ya que los sujetos interactúan con otros mientras van construyendo su identidad, participan de grupos y se comunican por medio lenguaje. Es decir, "el abordaje de las representaciones sociales nos permitiría acercarnos al modo en que los sujetos interpretan y construyen su conocimiento sobre la realidad" (Aguilar et. al., 2011, p. 3), sabiendo que éstas tienen un impacto en las actitudes y comportamientos.

Según Abric (1941-2012), uno de los autores que ha contribuido al desarrollo de esta teoría, las representaciones sociales constituyen un marco de referencia a partir del cual es posible describir, explicar y comunicar la realidad, favoreciendo los intercambios y la comunicación social (función del saber). Asimismo, hacen posible la construcción de una imagen de los grupos en sí mismos y en relación a los otros grupos (función identitaria) y "producen una anticipación que actúa como filtro de la interpretación de los acontecimientos" prescribiendo "las prácticas en contextos determinados" (función orientación). También "operan luego de la acción con el fin de perpetuar la posición del grupo" (citado por Aguilar et al., 2011, p. 3). Pese a esta complejidad, parafraseando a Jodelet (1985), teoría de las RS ha tenido alta valoración y aplicabilidad en diferentes disciplinas, cobrando especial sentido en las Ciencias Sociales y en Educación (Castorina, 2008, p. 9), al pretender una mayor comprensión de los fenómenos.

En sintonía con lo anterior, Anijovic y Cappelett (2017) precisan que "los profesores construyen representaciones sobre el aprendizaje y la enseñanza de modo intuitivo a través de la práctica profesional, mientras que, en el caso de los alumnos, estas son resultado de su propia experiencia como estudiantes" (Anijovic y Cappelett, 2017, p.40). De allí que las RS sean un constructo pertinente para explorar elementos implícitos del pensamiento del profesor que son relevantes de develar durante la Formación Inicial Docente, dado que la práctica del futuro se verá influida directa o indirectamente por un conjunto de significaciones y sentidos (representaciones) que ha construido al respecto durante su trayectoria formativa.

\section{Metodología}

El presente trabajo se enmarca en el estudio de casos (Pérez, 2011), sobre las representaciones de estudiantes de Pedagogía en Educación Básica en una universidad chilena del centro sur del país, pertenecientes a la cohorte 2017. Por tanto, no pretende generalizar resultados, sino más bien aportar antecedentes empíricos para una mejor comprensión de la Formación Inicial Docente. Dado que se busca indagar las representaciones de los sujetos en sus relatos, su naturaleza metodológica es hermenéutica. Es decir, se trata de un estudio de tipo descriptivo e interpretativo puesto que permite caracterizar las representaciones desde los propios actores o sujetos indagados (Goetz, y LeCompte, 1988; Eisner, 1998; Neiman y Quaranta, 2006). Por otro lado, desde el punto de vista de la tipología, corresponde a un estudio de tipo transversal, dado que la recolección de información se realizó una sola vez a todos los participantes.

\section{Participantes}

Según Patton (1988) la principal característica del muestreo cualitativo es su conducción intencional en búsqueda de casos ricos en información (en Quintana Peña, 2006, p. 57). En esta ocasión, se pretende recoger antecedentes en relación con las representaciones sobre la evaluación que han elaborado estudiantes en Formación Inicial Docente (FID) durante su preparación formal para la docencia, en cuyo período se supone cuentan con oportunidades formativas para vincularse al tema de la evaluación educativa como objeto de aprendizaje.

Específicamente, el grupo de interés son futuros profesores de Pedagogía Básica, quienes, al egresar y titularse, según las normativas vigentes, se encuentran habilitados para impartir docencia en todos los niveles de Enseñanza Básica, pudiendo impactar en un amplio rango de alumnos en el sistema escolar chileno. En coherencia, los participantes fueron estudiantes de Pedagogía en Educación General Básica de una universidad estatal y regional del centro sur de Chile, que cuenta con garantía de calidad al tener el $100 \%$ de sus carreras de Pedagogía acreditadas al año 2019, en conformidad a la Ley 20.129 (MINEDUC, 2006). Cabe precisar que ésta es la carrera más antigua del plantel y la única que, a nivel regional, incorpora una instancia formal de práctica pedagógica en sistema internado y en escuelas rurales durante el segundo ciclo formativo, lo que constituye un hito para los futuros profesionales; por lo cual resulta de interés explorar las representaciones sobre la evaluación que han construido antes de este momento.

Por tanto, en la selección de los sujetos se consideraron como condiciones de inclusión ser alumno regular de la cohorte 2017, que se encuentren con matricula vigente para el segundo semestre del 2018, 
REVISTA DE LA ESCUELA DE CIENCIAS DE LA EdUCACIÓN, AÑO 16, NRO. 15, VOL. 2, JULIO A DICIEMRE DE 2020. PÁGINAS 119-130. ISSN 2362-3349 (EN LÍNEA). REPRESENTACIONES SOBRE LA EVALUACIÒN DE ESTUDIANTES EN FORMACIÒN INCIAL DOCENTE ANA CAROLINA MALDONADO FUENTES. PEDRO RODRIGO SANDOVAL RUBILAR.

período que marca el fin del primer ciclo formativo. La nómina con datos de identificación de los estudiantes se obtuvo a partir de la ficha de antecedentes disponible en el sistema informático interno de la institución. La mayoría de los participantes son de género femenino $(86,27 \%)$ acorde a la tendencia nacional para las Carreras de Pedagogía Básica.

\section{Instrumento de recolección de información}

El instrumento consistió en una tarea de escritura de respuesta abierta, en la que los estudiantes (casos de estudio) debieron redactar un texto en prosa, de extensión libre, acerca de la evaluación como tarea docente. Las instrucciones y el formato de presentación de la tarea de escritura fueron sometidos a juicio de experto para su validación de contenido y coherencia entre el instrumento y el objeto pesquisado (Spindler y Spindler, 1987). Todos los participantes realizaron la tarea de escritura en el mismo momento y dentro del horario de trabajo presencial del grupo, bajo la supervisión de una única docente y con consentimiento informado (Meo, 2010).

\section{Análisis y procesamiento de la información}

De acuerdo con la naturaleza del estudio, se realizó un análisis de contenido de la información, para lo cual se elaboraron categorías y subcategorías emergentes, en torno a las cuales se agruparon las afirmaciones esgrimidas por los estudiantes acerca de cómo evaluar y para qué evaluar, incluyendo en este último caso algunas respuestas referidas a qué efectos tiene la evaluación. Un segundo análisis corresponde a los resultados descriptivos numéricos por unidad de análisis, por texto y por participante para cada una de las categorías y subcategorías. Para el procesamiento de la información se empleó el software de análisis cualitativo N-Vivo, versión 12; y, en lo que respecta a los resúmenes numéricos, se ocupó una planilla Excel; ambos con licencia de uso institucional.

A fin de contribuir a una mejor visualización de los datos, se tomaron algunas decisiones de escritura académica; a saber: para identificar las afirmaciones presentes en cada discurso se utilizará la terminología "Unidades de Análisis"; para la distinción de cada uno de los escritos de los estudiantes se hará uso de la noción de "Texto" y para la individualización de los textos según participantes se utilizarán números arábigos (p. e. 25, es el texto del estudiante 25). Asimismo, dada la naturaleza de los contenidos presentes en los discursos, cabe la posibilidad de que haya textos que contengan unidades de análisis alusivas a más de una categoría o subcategoría. En estos casos, el resultado final se expresa en una cuantificación mayor al número total de textos únicos (p. e. 17 en vez de 13). Cuando esto suceda, el dato se visualizará con la notación ${ }^{(*)}$ lo que significa que hay textos que se pueden contabilizar más de una vez en el total porque contienen unidades de análisis en una o más categorías o subcategorías (p. e. ${ }^{\left({ }^{*}\right)}$ se repite una vez; ${ }^{(* *)}$ se repite dos veces y ${ }^{(* *)}$ se repite en tres ocasiones. Ambos tipos de resultados se describen a continuación, siguiendo el orden de las preguntas orientadoras.

\section{Resultados}

Se presentan los resultados obtenidos por los sujetos evaluados en torno al objetivo de describir las representaciones sobre cómo y para qué se evalúa, a partir de los 51 relatos del corpus. En concreto, se describirán los datos agrupados según categorías, subcategorías y unidades de análisis, mediante resumen numérico y tablas de frecuencia.

\section{Representaciones sobre cómo evaluar}

A nivel global, de los 51 textos escritos, en 38 de ellos $(74,50 \%)$ se observan afirmaciones que responden "cómo evaluar"; dando origen a 4 categorías, 127 unidades de análisis independientes y 110 observaciones categorizadas. La distribución porcentual por categoría y texto se observa en la siguiente tabla:

Tabla 1. Distribución de respuestas por categoría respecto de Cómo evaluar

\begin{tabular}{lcccc}
\hline Categorías & \multicolumn{2}{c}{ Unidades de Análisis } & \multicolumn{2}{c}{ Participantes } \\
& Frecuencia & $\%$ & Frecuencia & $\%$ \\
\hline Instrumentos y procedimientos & 43 & 33,86 & 21 & 28,00 \\
Tipologías de evaluaciones & 32 & 25,20 & 17 & 22,67 \\
Evaluar de buen modo & 29 & 22,83 & 21 & 28,00 \\
Ajustado a variables de contexto & 23 & 18,11 & 16 & 21,33 \\
Total & 127 & 100,00 & 75 & 100,00 \\
\hline
\end{tabular}

Fuente: Elaboración propia 
De la tabla anterior se desprende que la mayoría de las respuestas de los participantes, un 59,06\%, se concentran en torno a categorías que aluden a clasificaciones que se encuentran documentadas en la literatura especializada, como instrumentos y procedimientos (33,86\%) y tipologías de la evaluación (ej: sumativa y formativa, diagnóstico, de proceso, autoevaluación o coevaluación) (25,20\%). Por tanto, los participantes asocian el cómo evaluar a la representación de cómo recolectar información, sin necesariamente distinguir el conocimiento de diferentes instrumentos de aquel que se refiere a tipologías de evaluación, lo que podría estar relacionado con experiencias previas de los sujetos participantes acerca de la evaluación durante su formación inicial.

Asimismo, un $22,83 \%$ de las unidades de análisis pormenorizan las características para evaluar "De buen modo" y un $18,11 \%$ hace referencia a que al evaluar habría que implementarlo "Ajustado a variables del contexto". A continuación, se describen los resultados en cada una de estas subcategorías, siguiendo el orden de la Tabla 1.

Tabla 2. Distribución de respuestas por categoría "Instrumentos y procedimientos"

\begin{tabular}{|c|c|c|c|}
\hline Subcategorías & Frec. & $\%$ & Participantes ${ }^{\text {(Repetido) }}$ \\
\hline Variedad de instrumentos y procedimientos & 18 & 41,86 & \\
\hline Pruebas & 11 & 25,58 & $\begin{array}{l}6^{(* *)}-12-19^{(* *)}-20-21^{(*)}-24 \\
25^{(*)}-26-27^{(* *)}-28-29^{(*)}-30\end{array}$ \\
\hline $\begin{array}{l}\text { Disertación/Exposición oral } \\
\text { Retroalimentación } \\
\text { Otras }\end{array}$ & $\begin{array}{l}07 \\
05 \\
02\end{array}$ & $\begin{array}{l}16,28 \\
11,63 \\
04,65\end{array}$ & $\begin{aligned} 31^{(* *)}- & 32^{(*)}-33^{(*)}-35^{(* *)}-37^{(*)} 38 \\
& -40-41^{(* \star)}-51^{(*)}\end{aligned}$ \\
\hline Total & 43 & 100,00 & 21 de 51 \\
\hline
\end{tabular}

Se infiere de la tabla anterior que la mayoría de los estudiantes ante la pregunta cómo evaluar se representan la idea de instrumentos. Así pues, del total de casos, un 25,58\% explicita el uso de pruebas, un $16,28 \%$ el de evaluar por medio de una disertación o exposiciones orales y un 11,63\% plantea la retroalimentación. Otros dos casos aluden a la observación o al uso de portafolio. En términos globales, los participantes responden con la representación de instrumento de recolección de información; además, se infiere la preponderancia de la prueba como representación principal.

Respecto a las tipologías de la evaluación, las respuestas se presentan en la Tabla 3.

Tabla 3. Distribución de respuestas por categoría "Tipologías de la evaluación"

\begin{tabular}{lccc}
\hline \multicolumn{1}{c}{ Subcategorías } & Frec. & $\%$ & Participantes \\
\hline Evaluación según agente & 11 & 34,38 & $1-2-12-13-18-20^{\left({ }^{(* *}\right)}-23$ \\
Momentos de la evaluación & 11 & 34,38 & $26-28-29^{(* \star)}-33^{(* *)}-35^{(* *)}$ \\
Funciones de la evaluación & 10 & 31,25 & $40-41^{(*)}-47^{(* *)}-48^{\left({ }^{(*)}\right.}-49^{(*)}$ \\
Total & 32 & 100,00 & 17 de 51 \\
\hline \multicolumn{2}{c}{ Obs. ${ }^{(")}$ se repite una vez ${ }^{(*)}$ se repite dos veces, ${ }^{(*)}$ se repite tres veces }
\end{tabular}

En relación con el "Agente Evaluador", cabe precisar que ningún caso explicita la idea de Heteroevaluación. En cambio, resalta que los participantes expresen la representación de Autoevaluación y algunos de ellos $(35,41$ y 48) también incorporan la Coevaluación. Al aludir a los "Momentos de la Evaluación", tres participantes lo asocian a la idea de la Evaluación Inicial, cinco a la Evaluación de Proceso y uno a la Evaluación Final. De ellos, cuatro incorporan también la noción de diagnóstico. Hay un caso (texto 20) que hace referencia a los tres momentos de la evaluación y hay otro (texto 33) que solamente vincula las evaluaciones inicial y final. Por último, solo cuatro sujetos aluden a las "Funciones de la Evaluación", dos con la representación de la Evaluación Formativa y Evaluación Sumativa (textos 47 y 49) y dos incorporan la Función Diagnóstica (textos 29 y 33). Es decir, ante la pregunta cómo evaluar, estos participantes lo asocian a la representación del estudiante como agente evaluador en un proceso que podría suceder en diferentes instancias, sin necesariamente coincidir en los momentos inicial, de proceso y final; o en las funciones formativa o sumativa. 
REVISTA DE LA ESCUELA DE CIENCIAS DE LA EdUCACIÓN, AÑO 16, NRO. 15, VOL. 2, JULIO A DICIEMRE DE 2020. PÁGINAS 119-130. ISSN 2362-3349 (EN LÍNEA). REPRESENTACIONES SOBRE LA EVALUACIÒN DE ESTUDIANTES EN FORMACIÒN INCIAL DOCENTE ANA CAROLINA MALDONADO FUENTES. PEDRO RODRIGO SANDOVAL RUBILAR.

A continuación, se presentan los datos en relación con lo que represetaría un buen proceso de evaluación, distinguiéndose subcategorías sobre el sujeto evaluador y las condiciones de ésta.

Tabla 4. Distribución de respuestas por categoría "Evaluar de buen modo"

\begin{tabular}{lccc}
\hline \multicolumn{1}{c}{ Subcategorías } & Frec & $\%$ & Participantes $^{\text {(Repetido) }}$ \\
\hline Docente "buen" evaluador & 13 & 44,83 & $1-5-6-18-20-21^{(*)}$ \\
Evaluación integrada al proceso de enseñanza & 06 & 20,69 & $23-24-27-28^{(*)}-29-30$ \\
& 05 & 17,24 & $34-35^{(*)}-36^{(*)}-37^{(*)}-38^{(* *)}$ \\
Condiciones emocionales & 05 & 17,24 & $39-40-42^{(*)}-46$ \\
Evaluación democrática & 05 & 21 de 51 \\
Total & 29 & 100,00 & 21 \\
\hline
\end{tabular}

Obs. ${ }^{(*)}$ se repite una vez ${ }^{(*)}$ se repite dos veces, ${ }^{(")}$ se repite tres veces

Tal como se observa en la tabla anterior, la mayor parte de los casos en esta subcategoría incorporan representaciones sobre la actuación esperada de un docente como "buen evaluador". A saber: es quien planifica la evaluación (6 casos), comparte las metas con sus estudiantes (3 casos), no se focaliza en el error (2 casos) y/o realiza una evaluación didáctica (2 casos). Por su parte, hay un grupo de sujetos que se representan la evaluación como integrada al proceso de enseñanza y aprendizaje, lo que equivale a $28,54 \%$ del total en esta categoría. Respecto de las condiciones emocionales, las ideas que resaltan son: por un lado, evaluar se relaciona con tener paciencia y respeto, generar confianza y promover un buen clima de aula; y, por otro, se espera que la evaluación sea evitando aburrir y sin generar miedo. Finalmente, cinco relatos, ante la pregunta cómo evaluar lo relación con la representación de una evaluación democrática, en el sentido de evaluar con participación activa de los estudiantes durante el proceso.

Al hacer referencia al contexto la evaluación, se desprenden las siguientes subcategorías:

Tabla 5. Distribución de respuestas por categoría "Ajustado a variables de contexto"

\begin{tabular}{|c|c|c|c|}
\hline Subcategorías & Frec. & $\%$ & Participantes ${ }^{\text {(Repetido) }}$ \\
\hline Evaluar según las características del estudiante & 09 & 39,13 & \\
\hline Evaluar según pruebas SIMCE-PSU & 03 & 13,04 & $4^{\left({ }^{*}\right)}-5-17-29-30^{(*)}-3235$ \\
\hline $\begin{array}{l}\text { Evaluar según los Estándares de Aprendizaje } \\
\text { Evaluar según las orientaciones MINEDUC } \\
\text { Evaluar según las orientaciones de UTP } \\
\text { Otras } \\
\text { Total }\end{array}$ & $\begin{array}{l}03 \\
02 \\
01 \\
05 \\
23\end{array}$ & $\begin{array}{c}13,04 \\
8,70 \\
4,35 \\
21,74 \\
100,00\end{array}$ & $\begin{array}{c}-36-37-39-41-42^{(* \star)} 46- \\
47-48-51^{(* *)}\end{array}$ \\
\hline
\end{tabular}

Obs. ") se repite una vez (") se repite dos veces, (") se repite tres veces

Se observa que gran parte de las representaciones sobre la idea de evaluar en forma contextualizada, incorporan aspectos asociados a las características del estudiante, lo que implicaría ajustarse a las condiciones individuales, tales como discapacidad, intereses, problemas personales (7 textos) o la situación social-cultural (2 textos). Por otra parte, ante la pregunta cómo evaluar, se responde asociando el proceso evaluativo a la representación de influencias externas al aula, como las pruebas del Sistema de Medición de la Calidad de la Educación (SIMCE) y la Prueba de Selección Universitaria (PSU) (3 casos), los Estándares de Aprendizaje (2 textos), las orientaciones del Ministerio de Educación de Chile (MINEDUC) y la Unidad Técnico Pedagógica (UTP). Es decir, la mayoría de los sujetos tiende a representar la evaluación como un proceso asociado a factores intervinientes, ajenos al mismo proceso evaluativo.

\section{Representaciones asociadas a para qué evaluar}

En general, en 40 de los 51 textos escritos $(78,43 \%)$ se observan afirmaciones que responden "para qué evaluar"; dando origen 5 categorías, 126 unidades de análisis independientes y 82 observaciones categorizadas. La Tabla 6 presenta la distribución porcentual por categoría y texto.

Tabla 6. Distribución de respuestas por categoría respecto de Para qué evaluar

\begin{tabular}{lcccc}
\hline Categorías & \multicolumn{2}{c}{ Unidades de Análisis } & \multicolumn{2}{c}{ Textos/Participante } \\
& Frecuencia & $\%$ & Frecuencia & $\%$ \\
\hline En función del aprendizaje & 63 & 50,00 & 30 & 36,59
\end{tabular}


En función de la calificación

En función del alumnado

En función del proceso de enseñanza-aprendizaje

En función del currículum

Total

$\begin{array}{cccc}23 & 18,25 & 18 & 21,95 \\ 14 & 11,11 & 12 & 14,63 \\ 14 & 11,11 & 11 & 13,41 \\ 12 & 09,52 & 11 & 13,41 \\ 126 & 100,00 & 82 & 100,00\end{array}$

Fuente: Elaboración propia

La tabla evidencia que el $50,00 \%$ de las unidades de análisis, extraídas de 30 textos independientes, se agrupan en torno a la categoría "En función del aprendizaje" (58,82\%). A continuación, se describen los resultados en cada una de las subcategorías, en el mismo orden de la presentación en la Tabla 6.

Tabla 7. Distribución de respuestas por categoría "En función del aprendizaje"

\begin{tabular}{|c|c|c|c|}
\hline Subcategorías & Frec & $\%$ & Participantes ${ }^{\text {(Repellao) }}$ \\
\hline Mejorar aprendizajes & 14 & 22,22 & \\
\hline Medir logro de aprendizaje & 13 & 20,63 & $1-7^{(7)}-13^{(7)}-19-23^{(7)}-25^{(7)}-26-27^{(7)}-28-29^{(7)}-30^{(7)} 31^{(7)}-$ \\
\hline Conocer aprendizajes adquiridos & 10 & 15,87 & $32^{(*)}-34^{(*)}-35-36^{(*)}-37^{(*)}-38^{(*)}-39^{(*)}-40^{(*)}$ \\
\hline Promover el aprendizaje efectivo & 08 & 12,70 & $\left(1^{*}\right)$ \\
\hline Reforzar aprendizajes & 07 & 11,11 & $41^{(7)}-42^{(7)}-43^{(7)}-44-45^{(7)}-46^{(7)}-47^{(7)}-48-49^{(7)}-51^{()}$ \\
\hline Verificar aprendizajes & 06 & 09,52 & \\
\hline Otros & 05 & 07,94 & \\
\hline Total & 63 & 100,00 & 30 de 51 \\
\hline
\end{tabular}

Al observar los resultados precedentes, un dato que resalta es que la mayoría de quienes responden con ideas "en función del aprendizaje" (23 textos, 76,77\% de estos casos) esgrimen argumentos también en otras subcategorías. Por ejemplo, hay 14 sujetos que plantean la idea de que "una evaluación se hace con el fin de lograr mejorar el aprendizaje" (texto 35) y un porcentaje de ellos, al mismo tiempo, afirma que se evalúa "para promover el aprendizaje efectivo" (texto 34 y texto 39) o "verificar aquellos conocimientos adquiridos por los alumnos" (texto 41). Esto indica que la pregunta se responde con conceptos "diversos" o desde una representación plurisignificativa; sin decantar una sola representación en torno a para qué evaluar.

En relación con la segunda subcategoría, en la Tabla 8 se presentan los resultados por frecuencia y textos-participantes.

Tabla 8. Distribución de respuestas por categoría "En función de la calificación"

\begin{tabular}{|c|c|c|c|}
\hline Subcategorías & Frec. & $\%$ & Participantes (Repetido) \\
\hline Calificar con notas & 13 & 56,52 & \\
\hline Categorizar a los sujetos & 04 & 17,39 & $\begin{array}{c}1^{(*)}-3-7-8-19-20-21-22-23^{\left({ }^{*}\right)}-24 \\
25^{(*)}-32-35-36-40^{(*)}-48-49^{\left(^{*}\right)}-51\end{array}$ \\
\hline $\begin{array}{l}\text { Otros } \\
\text { Total }\end{array}$ & $\begin{array}{l}06 \\
23\end{array}$ & $\begin{array}{c}26,09 \\
100,00\end{array}$ & 18 de 51 \\
\hline
\end{tabular}

Se desprende de la Tabla 8, que la mayoría de las respuestas en torno a para qué evaluar se asocia a ideas relacionadas con la representación de la calificación: "Calificar con notas" $(56,52 \%)$ y "Categorizar a los sujetos" (17,39\%). De los participantes que lo argumentan, 5 aportan ideas en más de un sentido y 13 coinciden en que esto se logra al asignar una nota (principal representación de la calificación para estos sujetos), lo que equivale a un $27,77 \%$ y $72,22 \%$ de los casos, respectivamente.

Otra posibilidad en la respuesta ante la pregunta para qué evaluar, se observó en función del currículum. La Tabla 9 resume los principales resultados al respecto.

Tabla 9. Distribución de respuestas por categoría "En función del currículum"

\begin{tabular}{lccc}
\hline \multicolumn{1}{c}{ Subcategorías } & Frec & $\%$ & Participantes \\
& & & (Repetido) \\
\hline Cumplir los objetivos del currículum & 6 & 50,00 & $3-4-6-11^{\left({ }^{*}\right)}-23-24$ \\
Medir contenidos predeterminados & 2 & 16,67 & $27-28-30-31-48$
\end{tabular}


REVISTA DE LA ESCUELA DE CIENCIAS DE LA EdUCACIÓN, AÑo 16, NRO. 15, VOL. 2, JULIO A DICIEMRE DE 2020. PÁGINAS 119-130. ISSN 2362-3349 (EN LÍNEA). REPRESENTACIONES SOBRE LA EVALUACIÒN DE ESTUDIANTES EN FORMACIÒN INCIAL DOCENTE ANA CAROLINA MALDONADO FUENTES. PEDRO RODRIGO SANDOVAL RUBILAR.

\begin{tabular}{lccc} 
Comparar con lo esperado & 2 & 16,17 \\
Otros & 2 & 16,17 \\
Total & 12 & 100,00 & 11 de 51 \\
\hline
\end{tabular}

La Tabla 9 informa la tendencia en torno a la idea de que se evalúa para "cumplir los objetivos del curriculum", siendo una representación presente en este grupo, dado que el 50,00\% de los casos (11 textos) argumenta en este sentido.

A continuación, en la Tabla 10 se presenta la distribución de respuestas para la subcategoría "En función del alumno", que recoge 12 textos, lo que equivale a un $23,53 \%$ del total de participantes del estudio.

Tabla 10. Distribución de respuestas por categoría "En función del alumno"

\begin{tabular}{|c|c|c|c|}
\hline Subcategorías & Frec. & $\%$ & Participantes ${ }^{\text {(Repetiao) }}$ \\
\hline Monitorear el progreso de los alumnos & 4 & 28,57 & $7^{(1)}-11-20-22-26-30$ \\
\hline Conocer el dominio del contenido del alumno & 2 & 14,29 & $31-32-34-35-39^{(*)}-43$ \\
\hline Evidenciar el desempeño de los alumnos & 2 & 14,29 & \\
\hline Otras & 6 & 42,86 & \\
\hline Total & 14 & 100,00 & 12 de 51 \\
\hline
\end{tabular}

En la tabla anterior, se destaca la representación de evaluar para "monitorear el progreso de los alumnos" (sobre el $25 \%$ de respuestas del grupo). Algunas afirmaciones que lo ejemplifican son: "para conocer el progreso (o en el peor de los casos el retroceso) que van teniendo nuestros alumnos" (texto 20), "ya que gracias a ella podremos conocer los avances obtenidos" (texto 30), o bien, "con la evaluación el docente va monitoreando el progreso del estudiante" (texto 31).

Estos resultados referidos a para qué evaluar se completan con la siguiente tabla:

Tabla 11. Distribución de respuestas por categoría "En función del proceso de e-a"

\begin{tabular}{lccc}
\hline \multicolumn{1}{c}{ Subcategorías } & Frec & $\%$ & Participantes \\
\hline Mejorar el proceso de e-a & 5 & 35,71 & \\
Oportunidad para mejorar la enseñanza & 3 & 21,43 & $13-20\left(^{* *}\right)-23-31\left(^{* * *}\right)-35$ \\
Tomar decisiones sobre el proceso de e-a & 2 & 14,29 & $38-41-45-46-47-49$ \\
Juzgar la enseñanza & 2 & 14,29 & \\
Otros & 2 & 14,29 & 11 de 51 \\
Total & 14 & 100,00 & \\
\hline
\end{tabular}

Obs. " se repite dos veces, (") se repite tres veces.

De la tabla anterior se desprende que las respuestas en esta subcategoría tienen resultados numéricos próximos, con frecuencias que fluctúan entre 2 y 5 alusiones. Sin embargo, a diferencia de lo sucedido en el resto de las subcategorías, acá hay sujetos que elaboran una respuesta integrando dos o tres ideas diferentes (texto 20 y texto 31), lo que daría cuenta de que la en la representación sobre para qué evaluar, hay coexistencia de conceptualizaciones al respecto.

Un dato de interés complementario se presenta en la Tabla 12, que sistematiza información respecto de cuáles son los efectos de la evaluación según los sujetos participantes del estudio.

Tabla 12. Distribución de respuestas para Efectos de la Evaluación

\begin{tabular}{|c|c|c|c|}
\hline Efectos de la Evaluación & Frec & $\%$ & Participantes $^{\text {(Repetido) }}$ \\
\hline $\begin{array}{l}\text { La evaluación expresada en calificación se comprende como } \\
\text { reflejo del aprendizaje }\end{array}$ & 11 & 20,37 & $\begin{array}{l}1^{(*)}-3-5^{(0)}-6-8- \\
10-11-1517^{(* * *)}\end{array}$ \\
\hline La evaluación sobrecarga, abruma o estresa a los estudiantes & 06 & 11,11 & $19^{(* * *)}-20^{(*)}-21^{(*)}-$ \\
\hline La evaluación se experimenta con énfasis en el resultado & 06 & 11,11 & $22^{(*)}$ \\
\hline La evaluación se asocia a aprendizaje por memorización & 06 & 11,11 & $24^{(*)}-25^{(*)}-28^{(*)}$ \\
\hline La evaluación etiqueta al estudiante (ej: flojo, inteligente) & 04 & 7,41 & $31-32^{(*)}-34^{(*)} 35^{(*)}$ \\
\hline La evaluación genera frustración en el estudiantado & 04 & 7,41 & $-36-3738^{(*)}-39-$ \\
\hline $\begin{array}{l}\text { La evaluación se experimenta como una competencia entre los } \\
\text { estudiantes }\end{array}$ & 03 & 5,56 & 40 42 $42^{(*)}-43-4546^{(*)}-$ \\
\hline La evaluación es un afán por superar la brecha & 03 & 5,56 & \\
\hline
\end{tabular}


aprendizajes

La evaluación impacta en la motivación o desmotivación por aprender

La evaluación a través de pruebas produce miedo o terror

Otros

Total

Obs. ${ }^{(")}$ se repite una vez ${ }^{(")}$ se repite dos veces, ${ }^{(")}$ se repite tres veces

3,70

3,70

12,96

100,00

Los resultados expresados en la Tabla 12, indican que la mayoría de las respuestas a la pregunta qué efectos tiene la evaluación, se hacen con ideas asociadas a representaciones negativas, tales como "sobrecarga", "miedo", "frustración" (12 casos); o bien, con ideas que representan un reduccionismo de la tarea de evaluar, al vincularla con "memorización", "competencia", o bien, "motivación o desmotivación" (11 casos). Además, son varios los casos que asocian la evaluación a las ideas de "evaluación como resultado", y se representa la calificación como "expresión de aprendizaje" (11 casos). Así pues, se evidencia que prevalece en los participantes ideas vinculadas a representaciones no necesariamente coincidentes entre sí.

\section{Discusión y conclusiones}

El presente estudio tuvo como finalidad caracterizar las representaciones respecto de la tarea de evaluar (cómo y para qué se evalúa) que han construido estudiantes de Pedagogía en Educación Básica durante su Formación Inicial Docente. Para ello, los participantes, como futuro profesor, redactaron textos al término del primer ciclo formativo, lo cual permitió contar con un corpus de 51 escritos y un conjunto de 307 unidades de análisis para responder cada una de las preguntas orientadoras del estudio.

De acuerdo al análisis de contenidos de sus respuestas e interpretación de las mismas, en primer lugar, se pudo inferir que, ante la pregunta cómo evaluar, no existe una representación común entre los sujetos, sino más bien coexisten diversos significados al respecto. Según la distribución, resaltan al menos tres sentidos en forma conjunta: cerca de un cuarto de las respuestas se asocia a representaciones sobre los instrumentos de recolección de información (23,86\%), otro cuarto a tipologías de la evaluación (25,20\%) y el restante $(50,94 \%)$ se refiere a las características que debe cumplir el proceso.

Este hallazgo podría explicarse en coherencia con el supuesto de que es posible identificar diferentes representaciones de la noción de evaluación cuando ésta se vincula al aprendizaje. Primero, pues en la literatura especializada se ha documentado que la noción de evaluación es un objeto de aprendizaje que puede conceptualizarse desde distintos enfoques; tales como: evaluación sumativa y evaluación formativa, evaluación normativa o evaluación criterial, la evaluación alternativa o evaluación auténtica, la evaluación del aprendizaje, para el aprendizaje , como aprendizaje, entre otros (Escudero, 2003; Popham, 2013; Santos Guerra, 2014; Moreno Olivos, 2014; Anijovich y Cappeletti, 2017; Förster, 2017). En segundo término, dado que autores como Feiman-Nemser (2008) afirman que la temática de la evaluación tiene el aspecto de un cuadro inconcluso, dado que el profesorado, generalmente, durante su formación y desarrollo profesional docente está en continuo proceso de aprendizaje en este ámbito.

Lo anterior, también podría estar relacionado con la evidencia de que existen múltiples modos de conceptualizar el aprendizaje y la enseñanza en los profesores, tal como lo han dado a conocer algunos estudios sobre teorías implícitas. Estos antecedentes invitan a reflexionar en torno a lo complejo que puede resultar, para los estudiantes en Formación Inicial Docente, disponer de claridad al momento de identificar los fundamentos de una u otra visión del aprendizaje, lo que podría incidir al momento de explicar el proceso de evaluación, tal como se ha evidenciado en este caso.

En segundo lugar, ante la pregunta para qué evaluar, se observa que existe una representación común en lo explicitado; y, que corresponde a los discursos dominantes del sistema escolar, dado que entre el $50 \%$ y $70 \%$ de las unidades de análisis lo asocia a la representación en función del aprendizaje o la calificación esperada. No obstante, al desglosar dichas categorías se constata una dispersión de significados en relación a lo representado por aprendizaje.

Una explicación podría estar en el predominio que ha tenido la función social y acreditadora de la evaluación a nivel de sistema educativo, dado que, en general, los documentos curriculares socializados para la gestión de los establecimientos escolares hacen referencia al cumplimiento de los Objetivos de Aprendizaje, tal como se observa en Bases Curriculares, Programas de Estudio, Estándares de Aprendizaje, entre otros (MINEDUC, 2018). Al mismo tiempo, es posible observar que hay un porcentaje alto de las respuestas que aluden a la idea de que la evaluación se representa como calificación, cuyo proceso consiste en asignar un código (número, letra o concepto) o escala numérica (Hawes, 2005); lo que, en otras palabras, corresponde a una transformación de los datos evaluativos recopilados por el profesor en información sobre el desempeño escolar de los estudiantes (Himmel et al., 1999).

Este tipo de resultado sería coincidente con Morales et al. (2008) quienes realizan un estudio de tipo cualitativo con profesores del sistema escolar colombiano y dan cuenta de una alta valoración de la 
REVISTA DE LA ESCUELA DE CIENCIAS DE LA EdUCACIÓN, AÑO 16, NRO. 15, VOL. 2, JULIO A DICIEMRE DE 2020. PÁGINAS 119-130. ISSN 2362-3349 (EN LÍNEA). REPRESENTACIONES SOBRE LA EVALUACIÒN DE ESTUDIANTES EN FORMACIÒN INCIAL DOCENTE ANA CAROLINA MALDONADO FUENTES. PEDRO RODRIGO SANDOVAL RUBILAR.

calificación como práctica evaluativa, bajo el supuesto de que ésta es una medida del conocimiento de los estudiantes. Asimismo, López Pastor y Palacios Picos (2012) hacen una distinción entre Prácticas de Evaluación Orientada a la Calificación (EOC) y las prácticas de Evaluación Orientada al Aprendizaje (EOA), afirmando que estas últimas son todavía minoritarias en la Formación Inicial del Profesorado. Más recientemente, Förster (2017) ha expuesto cómo el énfasis en la calificación ha tenido impacto en el sistema escolar chileno, dado que, por un lado, se solicita al docente que gestione los resultados de sus evaluaciones y tome decisiones sobre la enseñanza, pero, por otro, al pretender hacerlo, el monitoreo se realiza a partir de las calificaciones obtenidas, lo que constituye un dilema para los docentes.

De lo anteriormente señalado, se puede concluir que los participantes del estudio, como grupo social definido, no dispondrían de una representación común sobre cómo y para qué evaluar, independientemente de su formación. Es decir, aunque están sometidos a un mismo currículo y proceso formativo al interior de una misma institución formadora, los datos dejan en evidencia que no disponen de una representación común acerca de la evaluación como tarea docente. Ello podría implicar, a modo de hipótesis, lo siguiente:

Las representaciones sobre cómo y para qué evaluar se han construido previamente en su experiencia escolar, y habrían sido reforzadas por los procesos formativos experimentados en la FID, al menos en una parte del grupo. Dada la cantidad de subcategorías, es posible pensar que, parte del grupo ha podido resignificar algunas ideas, pero son tan diversas que no dan cuenta de un discurso dominante.

La Formación Inicial Docente no logra incorporar una nomenclatura única de la profesión, que permita de dotar de representaciones comunes del grupo social denominado profesor y, por ende, una identidad profesional con lenguaje compartido.

Se espera que los resultados contribuyan a fortalecer los procesos de Formación Inicial Docente, considerando escritos de los estudiantes, quienes durante su trayectoria formativa experimentan un proceso de cambio y profesionalización. En este sentido, se postula que es relevante conocer las representaciones acerca de la evaluación que han ido elaborando los estudiantes FID, puesto que en su futuro ejercicio docente serán los responsables de tomar decisiones sobre la evaluación de los aprendizajes.

\section{Referencias bibliográficas}

Agencia de Calidad de la Educación (2016). Estudio sobre Formación Inicial Docente en Evaluación Educacional. Santiago, Chile.

Aguilar, S. Mazzitelli, C.; Chacoma, M. y Aparicio, M. (2011). Saberes del docente y representaciones sociales: implicancias para la enseñanza de las ciencias naturales. En: Revista Electrónica Actualidades Investigativas en Educación, 11 (2), 1-28.

Ahumada, P. (2005). La evaluación auténtica: un sistema para la obtención de evidencias y vivencias de los aprendizajes. Revista Perspectiva Educacional, 45, 11-24.

Anijovich, R. y Cappeletti, G. (2017). La evaluación como oportunidad. Buenos Aires: Paidós. Voces de la Educación.

Barber, M. y Mourshed, M. (2008). Cómo hicieron los sistemas educativos con mejor desempeño del mundo para alcanzar sus objetivos. PREAL № 41, Buenos Aires, Argentina.

Black, P. y Wiliam, D. (1998). Assessment and Classroom Learning. En: Assessment in Education, 5 (1), $7-74$.

Camilloni, A. (1998). Calidad de programas e instrumentos de evaluación. En A. Camilloni, et. al. (Eds.) (1998). La evaluación de los aprendizajes en el debate didáctico contemporáneo. Paidós: Buenos Aires, Barcelona, Cuidad de México.

Cárcamo, H. (2015). Naturalización de los contenidos y objetivos de aprendizaje: acerca del énfasis que los futuros maestros de primaria ponen en los aspectos técnico-pedagógicos. En: Revista de Antropología Social, 24, pp. 271-285.

Castorina, J. (2008). (Comp.) Representaciones sociales. Problemas teóricos y conocimientos infantiles. Barcelona, España: Gedisa.

Comellas, M. (2000). La formación competencial del profesorado: formación continuada y nuevos retos organizativos. En: Revista Educar, 27, pp. 87-101.

Eisner, E. (1998). El ojo ilustrado: indagación cualitativa y mejora de la práctica educativa. Paidós: España.

Escudero, T. (2003). Desde los tests hasta la investigación evaluativa actual. Un siglo, el XX, de intenso desarrollo de la evaluación en educación. En: Revista Electrónica de Investigación y Evaluación Educativa, 9 (1), $11-43$

Feiman-Nemser, S. (2008). Teacher learning: How do teachers learn to teach? En M. Cochran-Smith, et. al. (eds.), Handbook of research on teacher education: Enduring questions in changing contexts (pp. 697-705). New York Routledge/Taylor \& Francis Group.

Ferry, G. (2016). Pedagogía de la formación. Buenos Aires, Argentina: Universidad de Buenos Aires; Ediciones Novedades Educativas.

Förster, C. (2017). (Ed). El poder de la evaluación en el aula. Mejores decisiones para promover aprendizajes. Santiago de Chile: Ediciones UC.

Goetz, J. y Lecompte, M. (1988). Etnografía y diseño cualitativo en investigación educativa. España: Morata S.L. 
Gutiérrez, E. (2016). Los docentes normalistas: representaciones sociales sobre formación docente. En: Lozano, I. y Gutiérrez, E. (2016). (Coord.) Procesos formativos y prácticas de los formadores de docentes (p.45-63). México D. F., México: Edic. Díaz de Santos.

Hawes, G. (2005). Evaluación de Competencias en la Educación Superior. Documento №12. Talca: Instituto de Investigación y Desarrollo Educacional, Universidad de Talca, pp.1-37.

Hernández-Nodarse, M. (2017). ¿Por qué ha costado tanto transformar las prácticas evaluativas del aprendizaje en el contexto educativo? Ensayo crítico sobre una patología pedagógica pendiente de tratamiento. En: Revista Electrónica Educare, 21(1), pp. 420-446.

Himmel, E.; Olivares, M. y Zabalza, J. (1999). Hacia una evaluación educativa. Aprender para evaluar y evaluar para aprender. Volumen I. Santiago, Chile: MINEDUC-Ediciones PUC.

Jackson, P. (1998). La vida en las aulas. Madrid, España: Morata.

Kelly, G. (1995). The Psychology of personal constructs. New York: Norton.

López Pastor, V. y Palacios Picos, A. (2012). Percepción de los futuros docentes sobre los sistemas de evaluación de sus aprendizajes. En: Teoría de la Educación. Educación y Cultura en la Sociedad de la Información, 13 (3), pp. 317-341.

Manzi, J.; González, R. y Sun, J. (2011). La evaluación docente en Chile. Ediciones Mide UC: Santiago, Chile.

$\mathrm{Meo}$, A. (2010). Consentimiento informado, anonimato y confidencialidad en investigación social. La experiencia internacional y el caso de la Sociología en Argentina. En: Aposta. Revista de Ciencias Sociales, 44, pp. 130.

Mercado Cruz, E. (2013). Acompañar al otro: saberes y prácticas de los formadores de docentes. Ediciones Díaz de Santos: México.

Ministerio de Educación (MINEDUC) (2018). Política para el fortalecimiento de la evaluación en aula. Santiago, Chile.

Ministerio de Educación de Chile (MINEDUC) (2006). Ley 20.129. Establece el Sistema Nacional de Aseguramiento de la Calidad de la Educación Superior. Santiago, Chile.

Morales, P.; Linares, P.; García, Á. y Corredor, J. (2008). ¿Qué pensamos cuando evaluamos? La evaluación al tablero. En: Enunciación, 13(1), pp. 38-44.

Moreno Olivos, T. (2014). Posturas epistemológicas frente a la evaluación y sus implicaciones en el currículum. Perspectiva Educacional. En: Formación de Profesores, 53(1), pp. 3-18.

Neiman, G. y Quaranta, G. (2006). Los estudios de caso en la investigación sociológica. En De Gialdino, Vasilachis (comp.), Estrategias de investigación cualitativa. Buenos Aires: Gedisa.

Pérez, D. (2011). La hermenéutica y los métodos de investigación en ciencias sociales. Estudios de Filosofía, (44), pp. 9-37.

Popham, J. (2009). Assessment Literacy for teachers: ¿faddish or Fundamental? Theory Into Practice, 48, pp. 411.

Quintana Peña, A. (2006). Metodología de investigación científica cualitativa. En Quintana, A. y Montgomery, W. (Eds.) (2006). Psicología: Tópicos de actualidad. Lima: UNMSM, pp. 47-84.

Salinas, M., Isaza, L. y Parra, C. (2006). Las representaciones sociales sobre evaluación de los aprendizajes. Revista Educación y Pedagogía, 18 (46), pp. 203-221.

Santos Guerra, M. (2014). La evaluación como aprendizaje. Cuando la flecha impacta en la diana. Madrid, España: Narcea.

Spindler, G. y Spindler, L. (1987). Interpretive ethnography of education: at home and abroad, Lawrence Erlbaum: Associates, Hillsdal, N. J.

Xu, Y. y Liu, Y. (2009). Teacher assessment knowledge and practice: A narrative inquiry of a Chinese college EFL teacher's experience. En: Tesol Quarterly, 43(3), 492-513. 